\title{
Protein Adsorption as a Key Mediator in the Nanotopographical Control of Cell Behavior
}

Elie Ngandu Mpoyi, ${ }^{\dagger, \S}$ Marco Cantini, ${ }^{*}, \dagger, \S$ Paul M. Reynolds, ${ }^{\dagger}$ Nikolaj Gadegaard, ${ }^{\dagger}$ Matthew J. Dalby, ${ }^{\dagger}$ and Manuel Salmerón-Sánchez ${ }^{* \dagger}$

${ }^{\dagger}$ Division of Biomedical Engineering, School of Engineering, University of Glasgow, Glasgow G12 8LT, United Kingdom "Center for Cell Engineering, Institute of Molecular Cell and Systems Biology, University of Glasgow, Joseph Black Building, University Avenue, Glasgow G12 8QQ, United Kingdom

\section{Supporting Information}

ABSTRACT: Surface nanotopography is widely employed to control cell behavior and in particular controlled disorder has been shown to be important in cell differentiation/maturation. However, extracellular matrix proteins, such as fibronectin (FN), initially adsorbed on a biomaterial surface are known to mediate the interaction of synthetic materials with cells. In this work, we examine the effect of nanotopography on cell behavior through this adsorbed layer of adhesive proteins using a nanostructured polycarbonate surface comprising $150 \mathrm{~nm}$-diameter pits originally defined using electron beam lithography. We address the effect of this nanopitted surface on FN adsorption and subsequently on cell morphology and behavior using C2C12 myoblasts. Wettability measurements and atomic force microscopy imaging showed that protein is adsorbed both within the interpits spaces and inside the nanopits. Cells responded to this coated nanotopography with the formation of fewer but larger focal adhesions and by mimicking the pit patterns within their cytoskeleton, nanoimprinting, ultimately achieving higher levels of myogenic differentiation compared to a flat control. Both focal adhesion assembly and nanoimprinting were found to be dependent on cell contractility and are adversely affected by the use of blebbistatin. Our results demonstrate the central role of the nanoscale protein interface in mediating cellnanotopographical interactions and implicate this interface as helping control the mechanotransductive cascade.

\section{A} wide variety of biological cells organize their surface structures in relation to the topographical features of the surface to which they adhere. ${ }^{1-5}$ Cells respond to growing on a nanostructured surface by changes in adhesion, cytoskeletal organization and other cellular activities, e.g., expression of a desired phenotype. ${ }^{1,5-8}$ It is believed that focal adhesions (FAs) underlie these cellular reactions to nanofeatures. For instance, focal adhesion size is altered in mesenchymal stem cells relative to underlying nanopatterned substrates: adhesion length and number decrease on highly ordered arrays allowing prolonged stem cell self-renewal and adding a small degree of controlled disorder increases adhesion size driving osteogenesis. ${ }^{9,10}$ Altering cellular adhesion produces changes in cytoskeletal organization and tension, which can affect cell behaviors. ${ }^{11}$ For example, platelet cytoskeletons have been shown to adapt to nanopitted surfaces with the shape of the features reflected in the actin microfilaments, ${ }^{2}$ and fibroblast actin filaments have been seen to rearrange similarly on nanoscale circles producing circular features in the cytoskeleton that were sensitive to integrin inhibition. ${ }^{12}$ This has been termed "nanoimprinting". ${ }^{2}$

A growing number of reports indicate that surface nanoarchitecture influences mechanotransduction, either through direct or indirect routes. ${ }^{11,13,14}$ Direct mechanotransduction describes the cell as a mechanical unit where conformational changes in the cell cytoskeleton alter the shape of the nucleus and consequently chromosomal arrangement and gene expression. On the other hand, indirect mechanotransduction describes biochemical signaling events. ${ }^{13}$ In response to surface features, for example, direct mechanotransduction can be driven by adhesion rearrangements changing cytoskeletal tension and arrangement and subsequently altering nucleus shape through linkers of nucleoskeleton and cytoskeleton (LINC) complexes. ${ }^{11}$ As adhesions have signaling molecules, such as focal

Received: March 8, 2016

Accepted: July 8, 2016

Published: July 8, 2016 


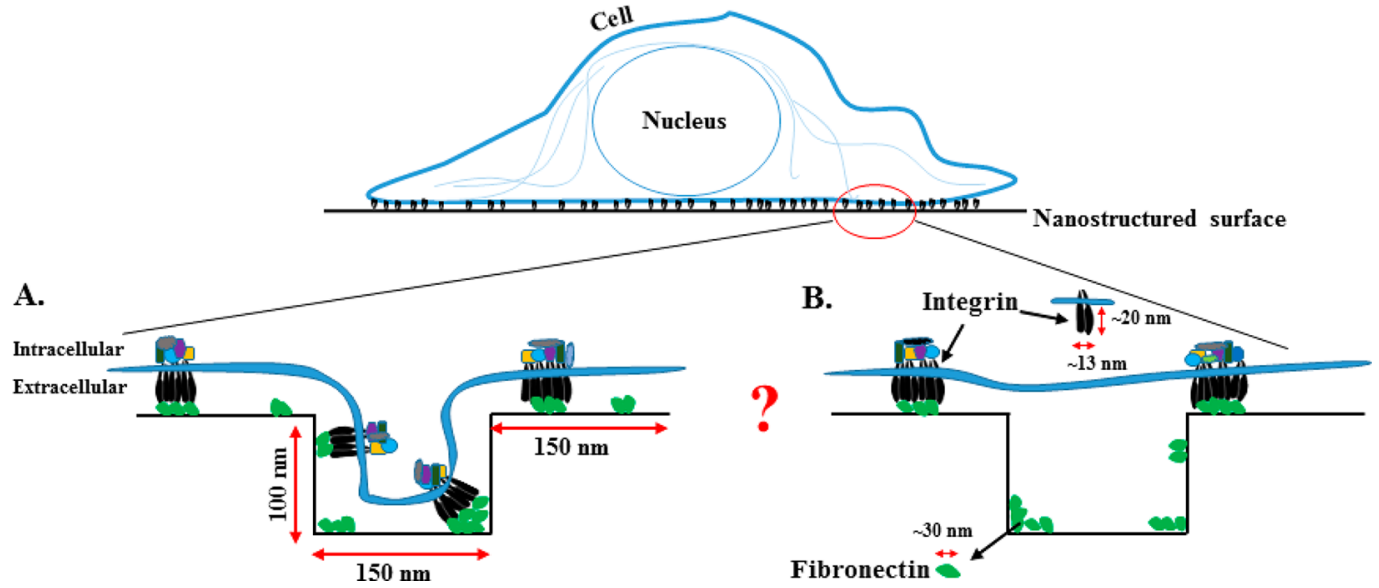

Figure 1. Proposed mechanisms for cell sensing of nanoscale features on a nanostructured surface. (A) Proteins (fibronectin) are adsorbed on the entire substrate surface, including the $100 \mathrm{~nm}$-deep nanopits, and cells are able to interact with the adsorbed protein through integrins, ${ }^{23,24}$ or (B) the nanopits are areas where the cells are not able to interact with the protein adsorbed at the bottom of the pits.

A.

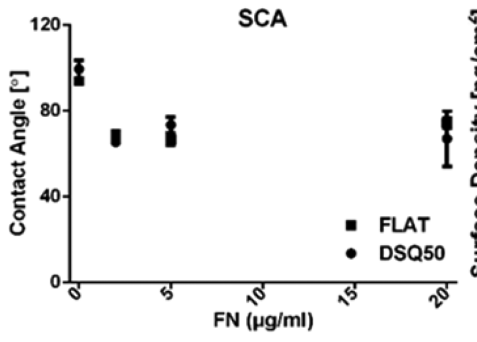

B.

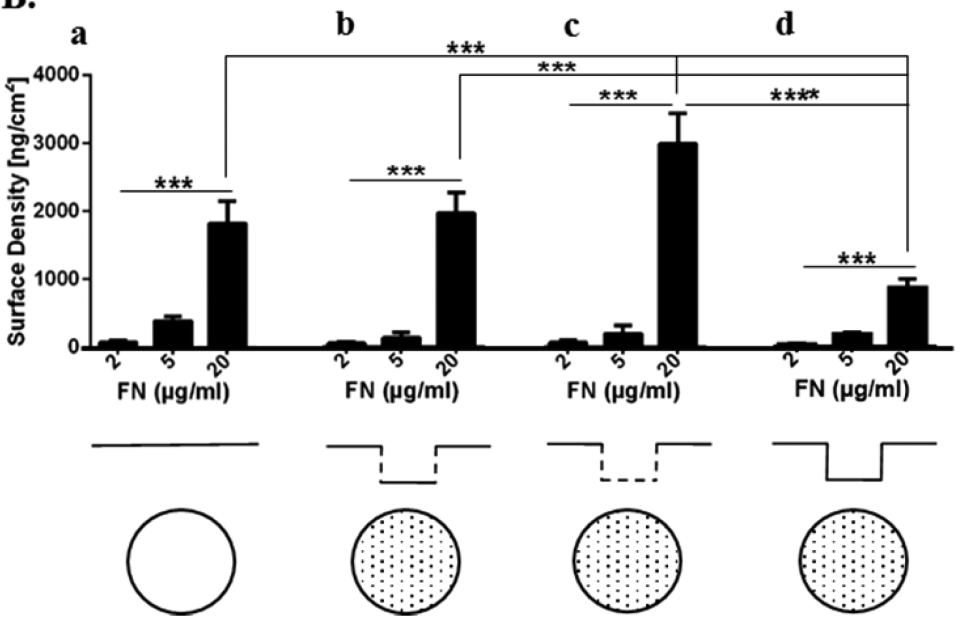

Figure 2. Characterization of FN adsorption on NSQ50 nanostructured surfaces. (A) Static water contact angle (SCA) measurements on NSQ50 (nanopits displaced randomly by $50 \mathrm{~nm}$ ) and flat (control) polycarbonate surfaces coated from 2,5 , and $20 \mu \mathrm{g} / \mathrm{mL} \mathrm{FN} \mathrm{solutions} \mathrm{for} 1$ h. (B) Quantification of surface density of adsorbed FN from the same FN solutions on a flat surface (area $\left.0.33 \mathrm{~cm}^{2}\right)(\mathrm{a})$, and on the NSQ50 nanostructured surface considering as areas available for adsorption either the projected area of the nanotopography $\left(0.33 \mathrm{~cm}^{2}\right)(\mathrm{b})$, the upper surface excluding the pits $\left(0.27 \mathrm{~cm}^{2}\right)(\mathrm{c})$, or the entire theoretical surface area $\left(0.51 \mathrm{~cm}^{2}\right)(\mathrm{d})$. Each figure represents an average of 3 samples per experiment and measurements were repeated 3 times. Values in graphs represent mean \pm standard deviation; statistically significant differences are indicated with $* * * p<0.001, * * * * p 0.0001$.

adhesion kinase (FAK), embedded within them, the same alterations lead to changes in G-protein and mitogen activated protein kinase activity that can have effects on spreading, growth and differentiation. ${ }^{11,15}$ However, the contribution of the protein interface is not well understood in cell-nanotopographical interactions.

It is recognized that cells do not directly interact with the material surface on which they are growing, but that their interaction depends on extracellular matrix (ECM) proteins for anchorage. The composition of this adsorbed protein layer is a key mediator of cell behavior. ${ }^{16-18}$ Nanotopographical features, being in the same size range as protein molecules, might have an effect on the amount, distribution and conformation of the adsorbed proteins that needs to be considered. ${ }^{19,20}$ Initial studies have demonstrated, for example, that nanoscale topography influences protein distribution between peaks and valleys, and this in turn controls cell adhesion altering the size of FA plaques, with larger FAs found on surfaces with an even distribution of the ECM protein fibronectin (FN). ${ }^{8,21}$ Selective protein nanoconfinement on different sized well-defined nanostructured surfaces has also been shown to correlate with cell behavior. ${ }^{22}$

To enhance our understanding of the interface between cells and nanotopography, we set to study the effect of a particular nanotopography implicated in cell differentiation on the adsorption of a main ECM component and subsequently on cell behavior. We used a surface consisting of disordered nanopits with $150 \mathrm{~nm}$ diameter arranged in a square pattern (300 nm center-center spacing) but with up to $\pm 50 \mathrm{~nm}$ offset from the center position. This surface, near-square 50 (NSQ50), has been previously shown to drive the osteogenic differentiation of mesenchymal stem cells. ${ }^{5}$ In this study, we use fibronectin, an abundant component of interstitial ECM and adhesive protein present in the serum usually used for cell cultures (including biomaterial/nanotopography research), ${ }^{5}$ and $\mathrm{C} 2 \mathrm{C} 12$ myoblast cells to study the mechanisms through 
FLAT PC
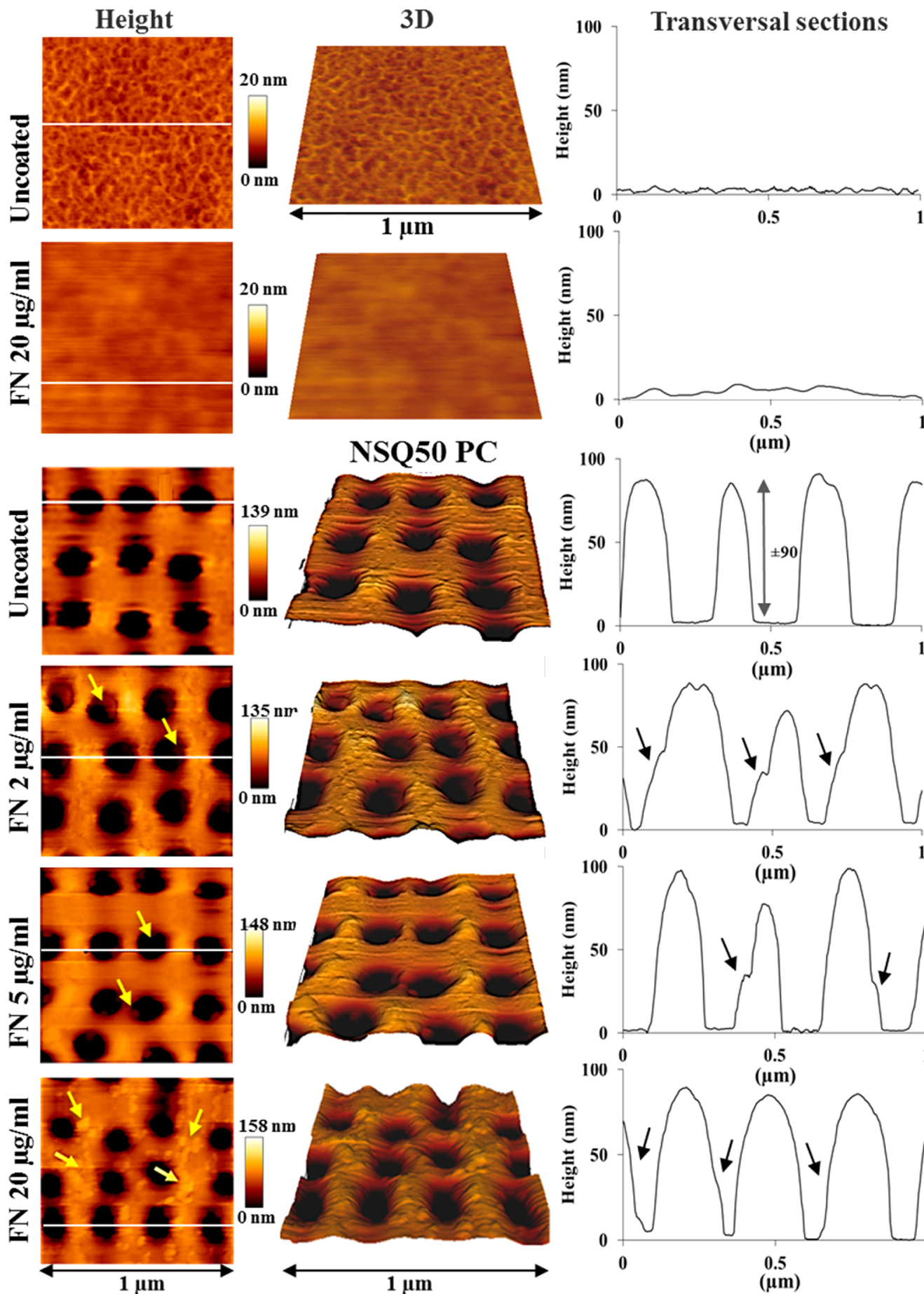
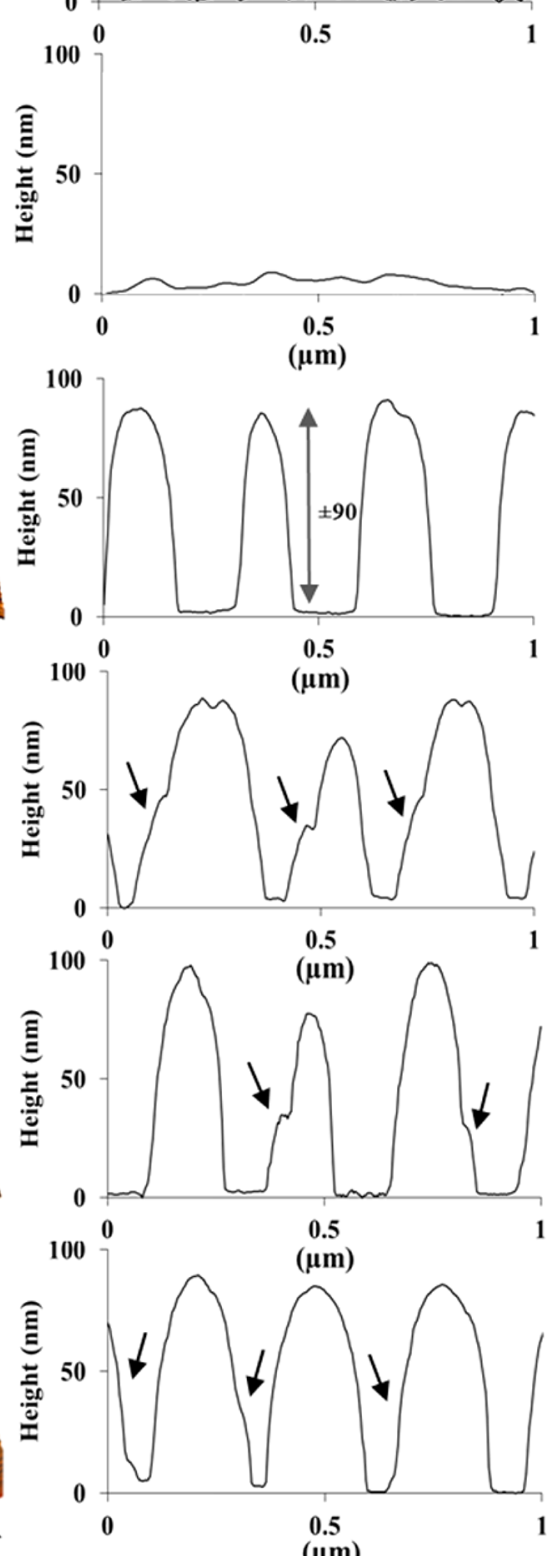

Figure 3. FN adsorption on the nanostructured surface. AFM images of flat control and NSQ50 nanotopography, either uncoated or coated with FN solutions of 2,5 , and $20 \mu \mathrm{g} / \mathrm{mL}$ for $1 \mathrm{~h}$. Height images (first column), 3D reconstruction of the surface (second column) and transversal section from the height images in correspondence of the white line (third column). Size $1 \mu \mathrm{m} \times 1 \mu \mathrm{m}$. Yellow arrows in the height images show examples of FN aggregates; black arrows in the sections show FN in the pits.

which cells sense nanotopographies (Figure 1). In particular, we want to know whether FN is adsorbed inside the nanopits and if the cells are able to exploit the FN molecules within them. FN adsorption onto the nanostructured surface was characterized in terms of amount and distribution inside and around the nanopits. The effect of this interface on $\mathrm{C} 2 \mathrm{C} 12$ cell adhesion and myogenic differentiation was then explored with particular focus on the cell cytoskeleton.

\section{RESULTS}

Fibronectin Adsorption on NSQ50 Nanostructured Surfaces. Figure $2 \mathrm{~A}$ shows the static contact angle measured on the nanostructured polycarbonate (PC) NSQ50 surface and on flat control before and after coating with $\mathrm{FN}$ for $1 \mathrm{~h}$ with solutions of concentrations 2,5 , and $20 \mu \mathrm{g} / \mathrm{mL}$. Overall, both surfaces were similar in terms of wettability: bare surfaces were 
hydrophobic $\left(94.0 \pm 2.1^{\circ}\right.$ for the flat surface and $99.5 \pm 4.1^{\circ}$ for the NSQ50) and became more hydrophilic once coated with FN $\left(\sim 70^{\circ}\right.$ for both surfaces), regardless of the concentration of FN (Figure 2A). Similar behavior was observed for dynamic measurements of wettability: the receding contact angles decreased to zero on both surfaces after FN coating, leading to high values of contact angle hysteresis, above $80^{\circ}$ on NSQ50 for concentrations greater than $5 \mu \mathrm{g} / \mathrm{mL}$ (Supplementary Figure S1). It is worth noting that the advancing angle was higher on uncoated nanostructured surfaces compared to flat controls, and the receding angle was lower, which is compatible with the enhancement of the wetting property of the surface as a function of its increase in surface area.

The surface density of adsorbed FN on the NSQ50 nanopits and flat surface was calculated by measuring the depletion of protein from solution. For FN solutions of 2, 5, and $20 \mu \mathrm{g} / \mathrm{mL}$ respectively, the density of FN adsorbed on NSQ50 and flat control surfaces similarly increased with solution concentration, from $\sim 100$ to $\sim 400$ and $\sim 1800 \mathrm{ng} / \mathrm{cm}^{2}$ on the flat surface, and from $\sim 50$ to $\sim 150 \mathrm{ng} / \mathrm{cm}^{2}$ and $\sim 2000 \mathrm{ng} / \mathrm{cm}^{2}$ on the nanostructured materials (Figure $2 \mathrm{~B}$ a, b). The density values were calculated considering three potential hypotheses. First, only the projected area of the surfaces adsorbs FN and if this were the case, no statistically significant differences would be detected between flat and nanostructured surfaces (Figure 2B b). Second, if it were the case that no protein could enter the nanopits, the density of adsorbed FN would be statistically higher on the NSQ50 surface compared to the flat control for a concentration of $20 \mu \mathrm{g} / \mathrm{mL}$ (Figure 2B c). Third, and conversely, if we considered the whole surface area as area available for FN adsorption, the density would be lower on the nanostructured surface (Figure $2 \mathrm{~B} \mathrm{~d}$ ).

To investigate these hypotheses further, nanostructured PC surfaces (bare and FN coated) were characterized by means of tapping mode atomic force microscopy (AFM). Figure 3 shows height images of the uncoated and coated flat surface (first and second row) and of the NSQ50 nanotopography, uncoated or coated with 2,5 , and $20 \mu \mathrm{g} / \mathrm{mL}$ FN solutions. The increase in surface area of the nanostructured surfaces compared to flat control measured via AFM ( $53 \%)$ was found to be in agreement with the theoretical value used to calculate the surface area $\left(0.51 \mathrm{~cm}^{2}\right)$ in Figure $2 \mathrm{~B} \mathrm{~d}$. Pits were shown to be $\sim 90 \mathrm{~nm}$ deep. The bare flat surface and the nanotopography, both the bottom of the pits and the top surface in between pits were very smooth, with a similar root-mean-square (RMS) roughness of $\sim 1.5 \mathrm{~nm}$ (Table 1 ). AFM images of other (ordered and disordered) bare nanotopographies are shown in Supplementary Figure S2. On the flat surface, FN is adsorbed in

Table 1. RMS Roughness of the Surfaces Calculated Using AFM $^{a}$

$\begin{array}{lcc}\text { nanotopographies } & \begin{array}{c}\text { surface RMS } \\ \text { roughness (nm) }\end{array} & \begin{array}{c}\text { nanopits (bottom surface) RMS } \\ \text { roughness (nm) }\end{array} \\ \text { FLAT } & 1.5 \pm 0.1 & \\ \text { NSQ50 } & 1.5 \pm 0.1 & 1.5 \pm 0.1 \\ \text { NSQ50 + FN2 } & 1.7 \pm 0.1 & 1.7 \pm 0.2 \\ \text { NSQ50 + FN5 } & 1.9 \pm 0.2 & 1.7 \pm 0.1 \\ \text { NSQ50 + FN20 } & 2.3 \pm 0.1 & 1.6 \pm 0.1\end{array}$

${ }^{a}$ Flat (FLAT) and nanostructured (NSQ50) PC surface before and after FN coating. FN2, $2 \mu \mathrm{g} / \mathrm{mL}$; FN5, $5 \mu \mathrm{g} / \mathrm{mL}$; FN20, $20 \mu \mathrm{g} / \mathrm{mL}$. aggregates and a continuous monolayer is formed upon FN adsorption from a $20 \mu \mathrm{g} / \mathrm{mL}$ solution (Figure 3, second row, and Supplementary Figure S3). On the nanostructured surfaces, after $\mathrm{FN}$ adsorption from a $2 \mu \mathrm{g} / \mathrm{mL}$ solution, protein was observed in globular aggregates both on the surface and inside the pits: transversal sections of the AFM height images showed the protein at the bottom of the nanopits (Figure 3, fourth row). Similar observations were made after adsorption from FN solutions of concentrations 5 and $20 \mu \mathrm{g} / \mathrm{mL}$; FN formed globular clusters on the surface and inside the nanopits.

More FN aggregates were spotted on the top surface of the nanotopographies increasing with direct proportionality to the concentration of the coating solution used and in accordance with data on FN density (Figure 2B). Interestingly, this was not the case for the inside of the nanopits, where fewer aggregates were seen at the higher FN concentrations $(20 \mu \mathrm{g} / \mathrm{mL})$. This was possibly due to high protein coverage of the bottom and walls of the pits from the adsorbed protein, as suggested by the decrease in pit depth (from $\sim 90$ to $\sim 80 \mathrm{~nm}$ ) and diameter (from $\sim 150$ to $\sim 100 \mathrm{~nm}$ ). Similar observations were made with other arrangements of nanostructured pits as shown in Supplementary Figure S4, showing protein adsorbed both in the interpits areas and inside the nanopits. Measurement of surface roughness confirmed the previous observations, as the roughness of the top surface of the nanotopography increased with FN solution concentration (Table 1). The increase of the roughness of the bottom of the pits was less pronounced and it was even diminished for the $20 \mu \mathrm{g} / \mathrm{mL}$ FN concentration, which is compatible with the formation of a layer of protein (Figure 3, sixth row). The same distribution of the protein, both inside the nanopits and outside of them, in the form of globular clusters, could be observed via scanning electron microscopy (SEM) (Figure 7A). The effect of the nanotopography on protein distribution was also confirmed by fluorescence microscopy of adsorbed FITC-labeled FN (Supplementary Figure S5); the technique lacked resolution to distinguish between bottom of the nanopits and spaces between them but, in accordance with the AFM results, a continuous protein layer was observed on the flat surface, whereas clusters organized by the nanofeatures were seen on NSQ50 polycarbonate. All the data illustrate that the whole surface area is available to FN adsorption and is thus indicative of a reduced FN density across the surface supporting the hypothesis proposed in Figure 2B d.

Cell Adhesion on NSQ50. Cell behavior on the NSQ50 nanotopography coated with FN was studied to assess the effect of FN-coated nanotopographies on $\mathrm{C} 2 \mathrm{C} 12$ myoblasts. Early adhesion experiments $(3 \mathrm{~h})$ were carried out in serum-free conditions with/without blebbistatin (inhibitor of myosin II) after coating the surfaces with a $20 \mu \mathrm{g} / \mathrm{mL}$ FN solution. $\mathrm{C} 2 \mathrm{C} 12 \mathrm{~s}$ were seeded at a low density $\left(5000\right.$ cells $\left./ \mathrm{cm}^{2}\right)$ on the samples to maximize cell-material interactions and to minimize cell-cell interactions.

Cells had a similar size on both flat and nanostructured surfaces in the presence or absence of blebbistatin (Figure 4A). To study the formation of FAs and quantify their maturation level on the NSQ50 nanostructured surface, frequency distributions of the FA size (defined as the length of the major axis of the FA plaque) were obtained through image analysis of the vinculin stained images (process detailed in Supplementary Figure S6, ${ }^{25,26}$ representative images of FAs in Figure 4F, G). FA complexes (dot-like complexes shorter than $1 \mu \mathrm{m}$ in size $)^{27}$ were discarded from the analysis. Fewer FAs 

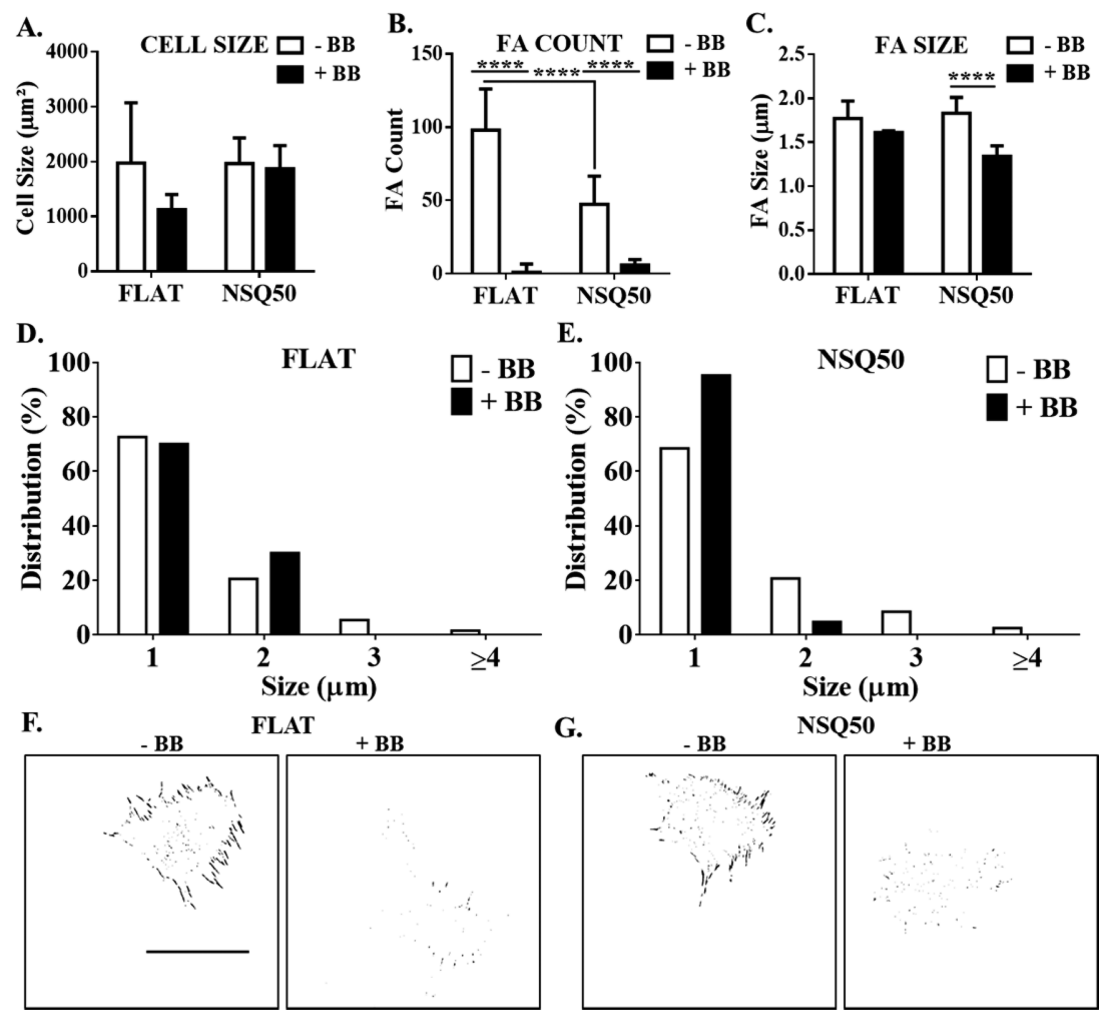

G.

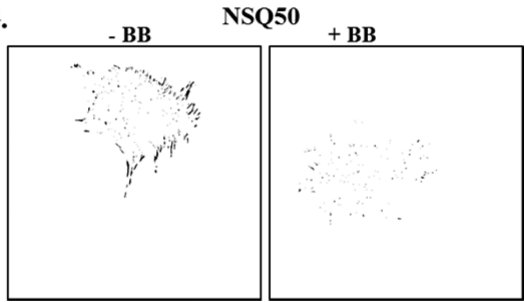

Figure 4. Focal adhesion quantification. Cell size (A), number of FAs per cell (B), average FA size (C), FA size distribution on flat (D) and NSQ50 (E) surfaces coated with FN at $20 \mu \mathrm{g} / \mathrm{mL}$ without and with blebbistatin (BB) in the culture medium. Graphs were quantified from images taken and processed through the FA server to build size distribution histograms of FAs. ${ }^{25}$ Representative inverted binary representation of FAs of $\mathrm{C2C12}$ cells on flat (F) and NSQ50 surface (G). Each figure represents mean \pm standard deviation from triplicate samples, repeated 3 times. Bar $50 \mu \mathrm{m}$. ****p $<0.0001$.
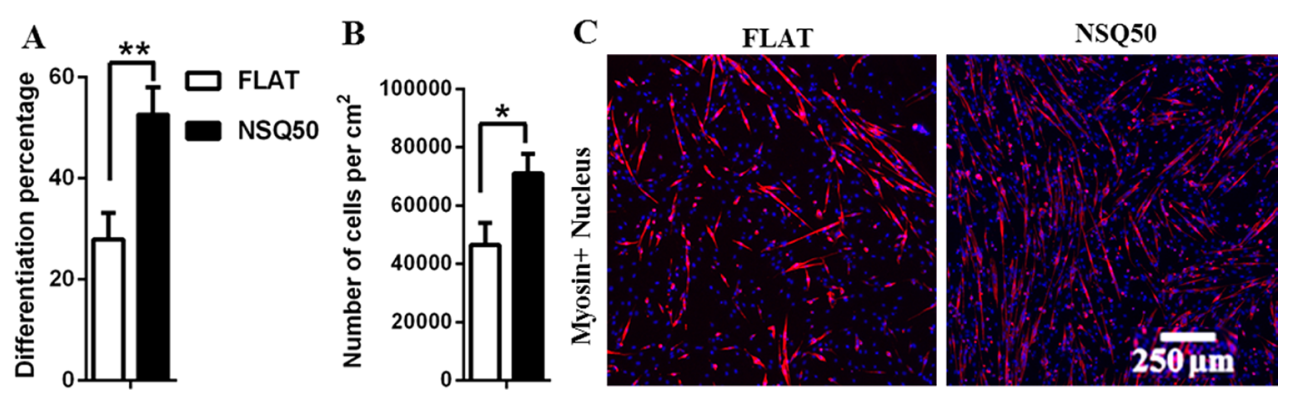

Figure 5. Cell differentiation and proliferation. (A) Degree of C2C12 cells myogenic differentiation on flat and NSQ50 surfaces coated with FN $20 \mu \mathrm{g} / \mathrm{mL}$ and (B) density of cells per $\mathrm{cm}^{2}$ after 4 days of culture. (C) Myosin labeled immunofluorescence images of C2C12 cells (red, sarcomeric myosin; blue, DAPI). Each figure represents mean \pm standard deviation from triplicate samples, repeated 3 times. Bar $250 \mu \mathrm{m} . * p$ $<0.05, * * p<0.01$.

were observed on the nanostructured surface compared to the flat control (Figure 4B). Moreover, the addition of blebbistatin significantly reduced the number of FAs on both surfaces. Size distribution was similar on nanostructured and flat polycarbonate, with a monotonic decrease from a higher fraction of smaller plaques (Figures 4D and E) and no significant difference in the average FA size (Figure 4C). On NSQ50 surfaces, a slightly higher percentage of long FAs $(\geq 3 \mu \mathrm{m})$ was observed ( $\sim 11 \%$ on NSQ50 vs $\sim 7 \%$ on control, Figure $4 \mathrm{D}$ and E); note that a shift from many smaller adhesion to fewer but larger adhesion has also been seen in osteoblasts ${ }^{28}$ and MSCs on this surface. ${ }^{5}$ Blebbistatin inhibited the formation of long FAs more significantly on the nanostructured surface compared to the flat control demonstrating tension dependence of the adhesions.
Cell Differentiation on NSQ50. Myogenic differentiation of $\mathrm{C} 2 \mathrm{C} 12$ cells grown on the NSQ50 nanopit topography was studied and compared to the flat control. The degree of differentiation was measured as the percentage of sarcomeric myosin-positive cells (Figure 5). Results showed significantly higher differentiation for cells on NSQ50 ( 50\%) compared to the flat control $(\sim 25 \%)$. Similarly, a higher number of cells were observed on the nanostructured surface. We note that cells did not proliferate on non-FN coated surfaces and that when a range of FN coated patterns were used no differences in cell growth were noted (Supplementary Figure S7).

Nanoimprinting of C2C12 Cells on NSQ50 Nanotopographies. To characterize the cell-FN-nanopit interaction, cell culture studies were performed in serum-free conditions and cells were observed via AFM. C2C12 cells were 

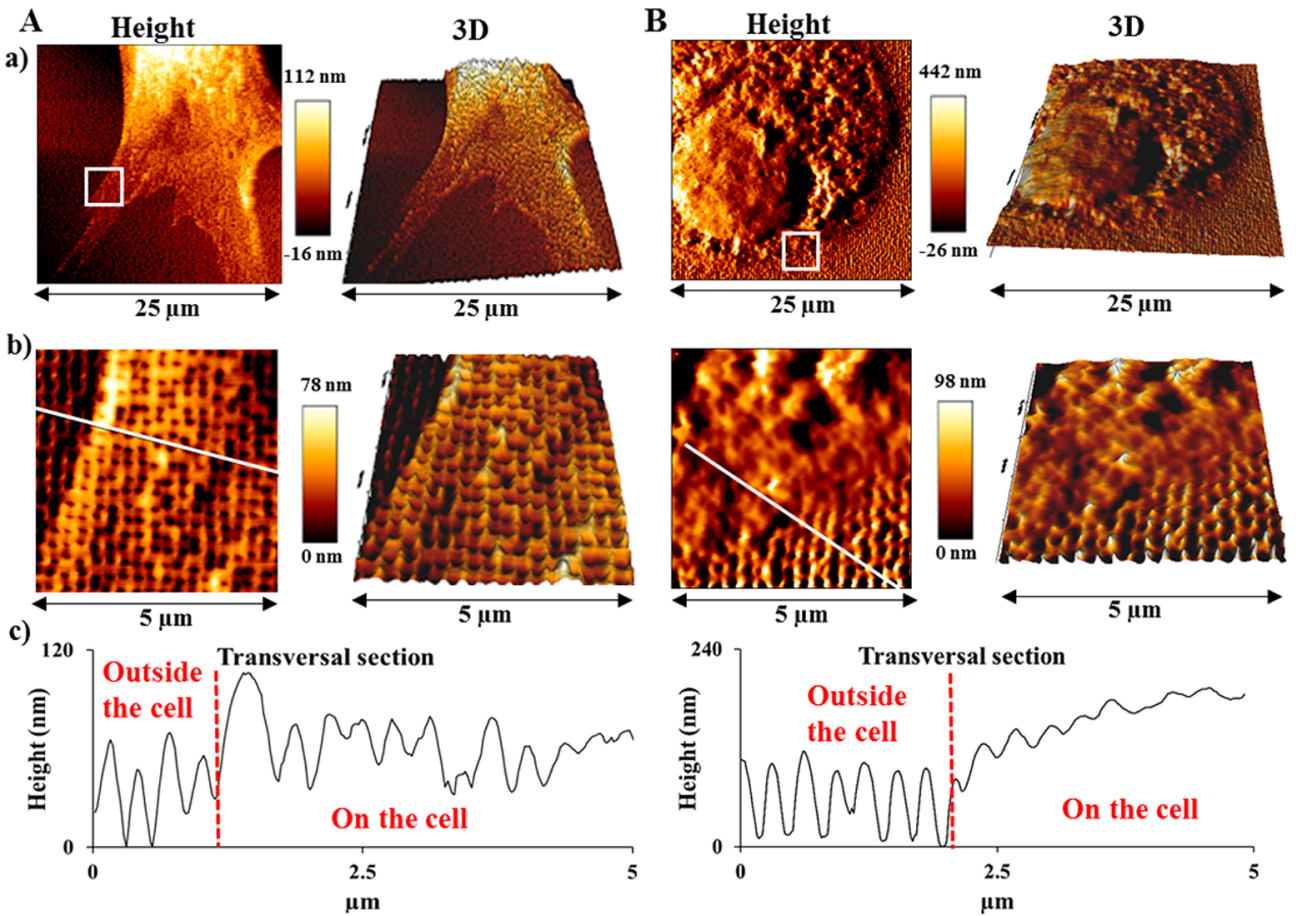
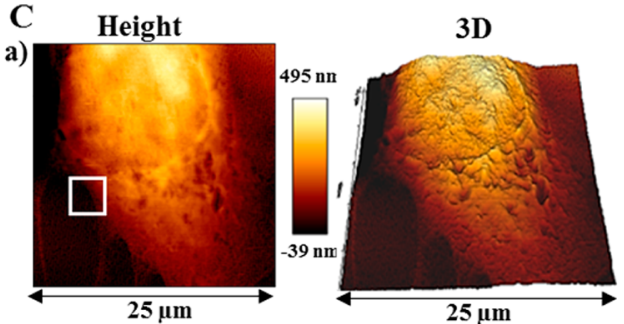

b)
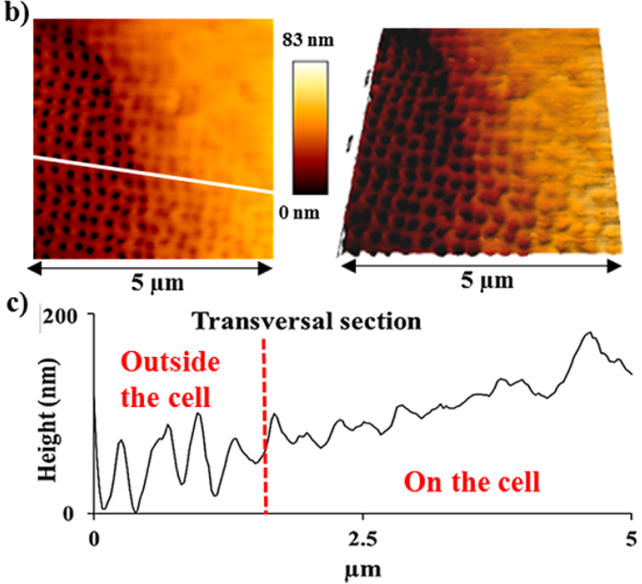
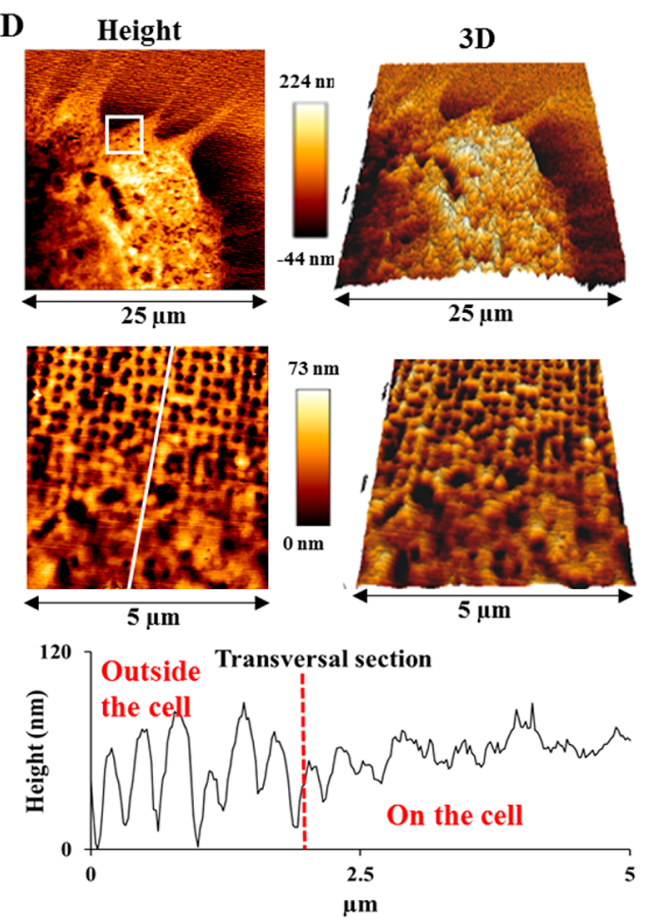

Figure 6. AFM images of $\mathrm{C} 2 \mathrm{C} 12$ cells. Cells grown on $\mathrm{NSQ} 50$ for $3 \mathrm{~h}$ in normal serum free medium after FN coating of the surface (A), on uncoated substrates (B), in medium containing blebbistatin (C), or paclitaxel (D) after FN coating of the nanotopography. In the first row (a) images $\left(25 \times 25 \mu \mathrm{m}^{2}\right)$ show the height magnitude and the $3 \mathrm{D}$ reconstruction of the cell. The second row $(\mathrm{b})$ shows image insets $\left(5 \times 5 \mu \mathrm{m}^{2}\right)$; the lower row (c) shows a transversal section of the cell-nanotopography interphase, corresponding to the white line in (b).

found to attach and spread on both $\mathrm{FN}$ coated flat and nanostructured surfaces (Figure 6A and Supplementary Figure S8A). Without any protein coating on the surface, cell spreading was impaired (Figure 6B). Furthermore, cells were able to spread on the protein coated surfaces in the presence of agents that interfere with cytoskeletal arrangement, namely blebbistatin (inhibitor of myosin II) or paclitaxel (microtubules stabilizer) (Figures 6C, D and Supplementary Figures S8B, C). AFM revealed differences in cell morphology across these various conditions. Cells grown in basal medium after FN coating of the surface spread and interacted with the nanopits; on the other hand, on uncoated nanostructures cells appeared to be more rounded and were not affected by the nanofeatures (Figure 6A, B). Cells treated with blebbistatin had a highly arborated morphology with thin membrane protrusions similar to retraction fibers. Finally, cells grown in the presence of paclitaxel were very well spread, with a bloated appearance and 
only small membrane protrusions (Figure 6C, D and Supplementary Figures S8B, C).

On the NSQ50 nanotopography, cells were seen to nanoimprint (Figure 6A a) and were noted to even protrude into the nanopits as highlighted by observing transversal sections at the cell edges (Figure $6 \mathrm{~A} \mathrm{~b}, \mathrm{c}$ ). On the other hand, in the absence of a protein coating cells appeared to reduce, almost completely, any nanotopographical interactions (Figure $6 \mathrm{~B} \mathrm{~b}, \mathrm{c})$. Moreover, during culture on FN coated nanotopographies, transversal sections demonstrated reduced ability of the cells to emboss the imprint of the substrate with blebbistatin or paclitaxel addition (Figure 6C and D); the extent of nanoimprinting was limited to the edges of the membrane protrusions. Scanning electron micrographs of cells grown on the nanostructured surfaces in the presence of a FN coating confirmed cell protrusions entering the nanopits (Figure 7B), further indicating the ability of cells to interact
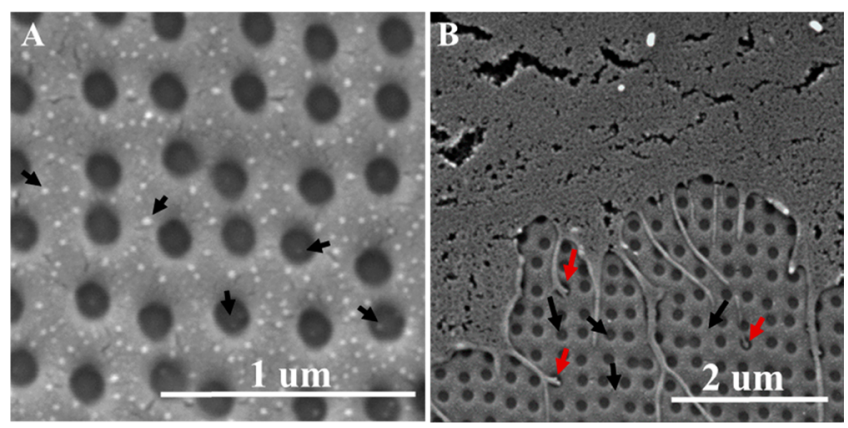

Figure 7. SEM images of NSQ50 nanotopographies. (A) Surface coated with a $20 \mu \mathrm{g} / \mathrm{mL}$ solution of $\mathrm{FN}$; arrows indicate examples of FN aggregates visible inside and outside the nanopits. (B) Edge of a $\mathrm{C} 2 \mathrm{C} 12$ cell adhered onto a nanotopography coated with FN; red arrows indicate examples of cell filopodia entering the nanopits, black arrows indicate examples of FN aggregates inside and outside the nanopits.

with FN molecules adsorbed at the bottom of the nanopits (Figure 7A). Through trialing adsorption of vitronectin, a multifunctional protein of the ECM that is, moreover, the other main cell adhesive component of serum, and culture with complete serum, cell nanoimprinting was found to be independent of the protein coating with cell filopodia entering the nanopits in all conditions (Supplementary Figure S9).

\section{DISCUSSION}

Various studies have demonstrated the influence of nanotopographies on protein adsorption ${ }^{8,21,22}$ and cell behavior $^{1-9,29-31}$ including stem cell differentiation ${ }^{5}$ and growth. ${ }^{4}$ These effects have been postulated to be transduced through indirect (biochemical) and direct (biomechanical) processes related to adhesion and cytoskeletal conformation. ${ }^{13}$ In this study, we aim to help elucidate the role of the protein interface and its involvement in the start point of the mechanotransductive cascade-adhesion formation and cytoskeletal reorganization (Figure 1).

Using a simple cell model, $\mathrm{C} 2 \mathrm{C} 12$ myoblasts, a difference in focal adhesion assembly and myogenic differentiation was observed comparing the NSQ50 nanotopography (which has been shown to trigger MSC osteogenic differentiation $)^{5}$ to flat controls in order to help show further potential of this surface. Previous studies have shown the role of $\mathrm{FN}$ in regulating $\mathrm{C} 2 \mathrm{C} 12$ behavior in vitro. ${ }^{32,33} \mathrm{FN}$ promotes $\mathrm{C} 2 \mathrm{C} 12$ alignment and fusion, and, in combination with nano- and microline patterns, enhances myogenic differentiation. ${ }^{32,33}$ Our results with the NSQ50 surface show no evident effect on C2C12 morphology or size. However, while the number of FAs diminished in cells on the nanotopography, the adhesions tended to become longer (Figure 4). This ties in well with formation of super mature adhesion on this surface in MSCs undergoing osteogenesis ${ }^{28}$ and with other reports showing that nanotopography can affect cell adhesion. ${ }^{5,8,9,34-37}$ The C2C12s were then noted to undergo more extensive myogenic differentiation (Figure 5). Importantly, the influence of the nanotopography was reversed by the addition of a contractility inhibitor, blebbistatin, in the culture medium (Figure 4). This indicates that the sensing of the nanoarchitecture is related to cell contractility. ${ }^{11}$ It is important to note that blebbistatin ablated the ability of cells to form large FAs more effectively on the nanotopography than on the flat controls demonstrating the importance of adhesion and cytoskeletal tension in cell response to the patterns.

In order to correlate these results to the adsorbed protein layer which mediates cell-material interaction, the material interface was characterized through wettability measurements, quantification of adsorbed $\mathrm{FN}$ and atomic force microscopy. Static and dynamic water contact angle measurements of the bare nanotopography revealed wetting of the entire surface area (Figures 2A and S1). Upon FN adsorption, both surfaces became more hydrophilic regardless of the amount of adsorbed protein. Unexpectedly, the potential increase in surface area granted by the nanotopography was not reflected in an increase of the amount of adsorbed protein. Both AFM and SEM analyses revealed that $\mathrm{FN}$ was adsorbed in globular clusters not only on the interpits spaces, but also inside the nanopits (Figure 3 and 7A), with higher coverage at increasing protein solution concentrations. This phenomenon was not limited to the NSQ50 nanotopography but occurred also on substrates with other arrangements of the nanostructured pits (Figure S4). The diameter of the pit $(150 \mathrm{~nm})$ appeared to be large enough to avoid nanoconfinement effects ${ }^{22}$ and to allow $\mathrm{FN}$ to be adsorbed both at the bottom and on the walls of the nanopits (Figure 3). This means that the entire surface area of the nanotopography was available for FN adsorption, as suggested by the wettability data. As a result, when FN was adsorbed from a $20 \mu \mathrm{g} / \mathrm{mL}$ solution, surface density values on the nanoarchitecture were reduced compared to the flat control. This is in agreement with the hypothesis proposed in Figure 2B d. As on the nanopits the FN conformation tended to be globular and confluent adsorption was not noted (as on flat control at $20 \mu \mathrm{g} / \mathrm{mL}$, Figures S3 and S5), we can postulate that the nanotopographical features, being in the same size range as protein molecules, potentially disrupt $\mathrm{FN}$ adsorption. This disruption may act to reduce the continuous protein coverage observed on flat controls, resulting in a less efficient FN adsorption per unit of surface area.

Nanoimprinting ${ }^{2,34}$ was observed in the $\mathrm{C} 2 \mathrm{C} 12$ cells grown on the nanotopography coated with $20 \mu \mathrm{g} / \mathrm{mL}$ of FN (Figure 6A). Many types of cells use filopodia to probe their nanoenvironment and filopodia have been seen to interact with nanoscale topographies. ${ }^{1,35}$ Here, however, is the evidence that cells can probe inside such small features. The C2C12s exhibited filopodia that made contact with the edges of pits and also entered the nanopits (Figure 7B). The reorganization of adhesions and alterations in cell "sensing" led to embossing of the nanopit morphologies into the membranes of the cells. On 
the other hand, no evidence of interaction of the cells with the nanofeatures was found when the surface was not coated with fibronectin, pointing to a critical role of the protein interface (Figure 6B).

Inhibition with blebbistatin caused disruption of nanoimprinting suggesting that adhesion-based cytoskeleton tension drove the effect as cells interacted with the protein coated features (Figure 6C). Stabilizing the cytoskeleton with paclitaxel also inhibited the nanoimprinting effect suggesting that the cytoskeleton needs to be dynamic to adapt to the feature morphologies (Figure 6D). Furthermore, nanoimprinting was independent of the protein coating and occurred in the presence of other ECM proteins besides FN (Figure S9). Critically we show that while the effect is dependent upon nanoscale topography, it is mediated by the protein layer and without this layer, the effect is not observed. This demonstrates that the biointerface is a critical component of the nanotopography-induced mechanotransduction.

\section{CONCLUSION}

In this work we show that many of the topography-driven changes in adhesion and cytoskeleton organization responsible for processes such as the control of cell differentiation are mediated by the protein interface. Furthermore, we show that FN can coat the insides of nanoscale pits and facilitate cell exploration of the pits.

\section{METHODS}

Polycarbonate Substrates. Nanotopographic pits patterns were manufactured in polycarbonate (PC) using a 3-step process: electron beam lithography, ${ }^{38}$ nickel die fabrication and injection molding. In short, the master substrates were fabricated to form an array of $150 \mathrm{~nm}$ diameter pits of $100 \mathrm{~nm}$ depth and $300 \mathrm{~nm}$ average pitch in a disordered square array with dots displaced randomly by up to $50 \mathrm{~nm}$ on both axes from their position in a square lattice (NSQ50). A flat PC surface was used as control.

Protein Adsorption. The model surfaces (NSQ50 and flat) were cleaned with distilled water, dried and coated with human plasma FN (Sigma) solutions at 2, 5, and $20 \mu \mathrm{g} / \mathrm{mL}$ in Dulbecco's phosphate buffered saline (DPBS) for $1 \mathrm{~h}$.

Water Contact Angle. Water contact angle (WCA) analysis was carried out on bare PC substrate and on FN-coated surfaces. Measurements were carried out using the sessile drop method with a Theta optical tensiometer (Biolin Scientific, Stockholm, Sweden). For each condition, the static contact angle (SCA) of the drop was determined by placing a drop of $3 \mu \mathrm{L}$ of Milli-Q water on the surface and measuring the angle of the drop with the substrate surface. Also, advancing contact angle (ACA) and receding contact angle (RCA) were determined by changing the volume of the droplet (respectively adding or removing water from the previously deposited drop) and measuring the contact angle when the three phase boundary between water, air and substrate moved. All these measurements were performed at room temperature and average values were obtained from 3 measurements of at least three different samples.

Quantification of Adsorbed FN. The biochemical assay based on the bicinchoninic acid assay (BCA protein assay, Thermo Fisher Scientific, Waltham, MA) was used following manufacturer instructions to determine the total amount of protein adsorbed on the PC nanotopography substrate surfaces. The latter were coated with FN for $1 \mathrm{~h}$ at different solution concentrations and the density of adsorbed protein was determined by measuring the amount of nonadsorbed FN. After coating for $1 \mathrm{~h}$, the FN solution was collected and transferred to a 96-well plate followed by the addition of the bicinchoninic acid working reagent (Thermo Fisher Scientific, Waltham, MA). FN solutions at 20,5 , and $2 \mu \mathrm{g} / \mathrm{mL}$ were used as standards. The plate was then placed in an incubator for $2 \mathrm{~h}$ at $37^{\circ} \mathrm{C}$. The absorbance was read on a Tecan NanoQuant Infinite M200 Pro plate reader (Männedorf, Switzerland) at $562 \mathrm{~nm}$ and the total protein adsorbed on the substrates was calculated subtracting the amount of protein remaining in the supernatant from the total amount of protein in the initial solution.

Cell Culture. Mouse C2C12 myoblasts (American Type Culture Collection, Blau et al. 1985) were maintained in growth media (Dulbecco's modified Eagle's media with $4.5 \mathrm{~g} / \mathrm{L}$ glucose and Lglutamine, Gibco) supplemented with $20 \% \mathrm{v} / \mathrm{v}$ heat inactivated fetal bovine serum (FBS, Gibco), and $1 \% \mathrm{v} / \mathrm{v}$ penicillin/streptomycin solution $(\mathrm{P} / \mathrm{S}, \mathrm{Gibco})$ after thawing and passaged using standard techniques. The cells were passaged by trypsination $(0.5 \%$ trypsin in $0.5 \mathrm{mM}$ EDTA, Gibco) from the culture flask at $70 \%$ confluence.

Atomic Force Microscopy. AFM experiments were performed with a Nanowizard 3 (JPK, Berlin, Germany). The images were acquired in tapping mode in air using silicon cantilevers (FMV from Bruker AFM Probes, Billerica, MA) with a pyramidal tip, a force constant of $3 \mathrm{~N} / \mathrm{m}$, a resonance frequency of $\sim 75 \mathrm{kHz}$, and a radius of curvature of $10 \mathrm{~nm}$. A schematic of the cantilever tip and of its size in relation to the nanotopographical features of the surface is shown in Supplementary Figure S10. Height, phase and amplitude magnitudes were recorded simultaneously for each image. Images were analyzed using JPK processing software and Gwyddion (64 bit) to calculate the average depth of the pits of the nanotopographies, their surface area and roughness.

To do AFM experiments on cells, cells were seeded on the samples at 5000 cells $/ \mathrm{cm}^{2}$ in media with or without $10 \mu \mathrm{M}$ blebbistatin (BB) or $10 \mu \mathrm{M}$ paclitaxel and were fixed with formaldehyde $3.7 \%$ for $20 \mathrm{~min}$ at $4{ }^{\circ} \mathrm{C}$ after incubation for $3 \mathrm{~h}$ without FBS. The samples were washed with DPBS, then water, finally dried with an air flow.

Scanning Electron Microscopy. C2C12 murine myoblast cells were seeded on the samples at 5000 cells $/ \mathrm{cm}^{2}$ and were fixed in $1.5 \%$ glutaraldehyde/ $/ .1 \mathrm{M}$ sodium cacodylate buffer for $1 \mathrm{~h}$ at $4{ }^{\circ} \mathrm{C}$. Then, cells were washed 3 times in $0.1 \mathrm{M}$ sodium cacodylate buffer before a 1 -h incubation in $1 \%$ osmium tetroxide/0.1 M sodium cacodylate buffer. Samples were then washed 3 times with distilled water and stained with $0.5 \%$ uranyl acetate/distilled water for $1 \mathrm{~h}$ in the dark. Samples were washed again with distilled water before dehydration through an ethanol gradient (30, 50, 70, 90 and 100\% ethanol). Finally, samples were loaded onto a POLARON E3000 Critical Point Dryer $\left(\right.$ Liquid $\mathrm{CO}_{2}$ ) for $1 \mathrm{~h} 20 \mathrm{~min}$ and then given a gold/palladium coating using a POLARON SC515 SEM COATER and viewed on a JEOL6400 SEM running at 10 kilovolts.

Cell Adhesion. $\mathrm{C} 2 \mathrm{C} 12$ cells were seeded at 5,000 cells $/ \mathrm{cm}^{2}$ density in media $\pm 10 \mu \mathrm{M} \mathrm{BB}$ on the nanotopography substrates (sterilized under UV for $20 \mathrm{~min}$ in 4 wells plates) previously coated for $1 \mathrm{~h}$ with FN $20 \mu \mathrm{g} / \mathrm{mL}$. Cells were fixed with formaldehyde $3.7 \%$ for $20 \mathrm{~min}$ at $4{ }^{\circ} \mathrm{C}$ after incubation for $3 \mathrm{~h}$ without FBS. The samples were permeabilized for $5 \mathrm{~min}$ using a Triton X-100 based permeabilization buffer $(0.5 \% \mathrm{v} / \mathrm{v}$ Triton X-100, $10.3 \% \mathrm{w} / \mathrm{v}$ saccharose, $0.292 \% \mathrm{w} / \mathrm{v}$ $\mathrm{NaCl}, 0.06 \% \mathrm{w} / \mathrm{v} \mathrm{MgCl} 2$, and $0.476 \% \mathrm{w} / \mathrm{v}$ HEPES adjusted to $\mathrm{pH}$ 7.2 ), and blocked with DPBS/BSA $1 \% \mathrm{w} / \mathrm{v}$ for $30 \mathrm{~min}$. Then samples were stained for $1 \mathrm{~h}$ at room temperature (RT) with a primary antibody against mouse vinculin hVIN-1 (Sigma-Aldrich, St. Louis, $\mathrm{MO}$ ), which stains the FAs. Afterward, the samples were washed 3 times with DPBS/tween $200.5 \% \mathrm{w} / \mathrm{v}$, then incubated for a further $1 \mathrm{~h}$ in the dark at RT with a secondary antibody (Cy3 antimouse), coupled with BODIPY FL phallacidin (Invitrogen) which stains the actin filaments. Finally, the samples were washed 3 times and then mounted with Vectashield containing DAPI (Vector Laboratories, Peterborough, UK) before visualizing using an epifluorescence microscope. Images were taken and channels merged using Image $(1.47 \mathrm{v})$ to localize nuclei, actin, and FAs. The latter were quantified using the FA analysis server. ${ }^{25}$ Image $(1.47 \mathrm{v})$ was also used to analyze immunostained pictures.

Myogenic Differentiation Experiment. $\mathrm{C} 2 \mathrm{C} 12$ cells were harvested and seeded at 20000 cells $/ \mathrm{cm}^{2}$ in DMEM supplemented with $1 \% \mathrm{P} / \mathrm{S}$ and $1 \%$ insulin-transferrin-selenium-X (Life Technologies) on the NSQ50 and flat surfaces previously coated with FN 20 $\mu \mathrm{g} / \mathrm{mL}$ for $1 \mathrm{~h}$. Medium was changed after $3 \mathrm{~h}$ and again after 2 days. 
The cells were kept for 4 days at $37{ }^{\circ} \mathrm{C}$ in a humidified atmosphere under $5 \% \mathrm{CO}_{2}$ before fixing with a 20:2:1 mixture of $70 \%$ ethanol, $37 \%$ formaldehyde and acetic acid. Samples were blocked for $1 \mathrm{~h}$ at RT with $5 \%$ goat serum in DPBS and stained for sarcomeric myosin by incubating with primary antibody (MF-20, Developmental Studies Hybridoma Bank) 1:250 at $37^{\circ} \mathrm{C}$ for $1 \mathrm{~h}$; afterward the samples were once more blocked for $10 \mathrm{~min}$ at RT, and then the secondary antibody (rabbit antimouse Cy3, Jackson Immunoresearch) 1:200 was added at $37^{\circ} \mathrm{C}$ for $1 \mathrm{~h}$. Samples were finally mounted on microscopy slides with mounting medium containing DAPI to stain the nuclei (VectaShield with DAPI, Vector Laboratories). All the samples were in triplicates. Images were acquired with an inverted Zeiss Axio Observer Z1 microscope.

Statistical Analysis. For the comparison of the different groups, one-way ANOVA was used with a Bonferroni post hoc test to compare all columns (GraphPad Prism 5.03) and the differences between groups were considered significant for $p<0.05$. All error bars are standard deviation.

\section{ASSOCIATED CONTENT}

\section{S Supporting Information}

The Supporting Information is available free of charge on the ACS Publications website at DOI: 10.1021/acsnano.6b01649.

Water contact angles measurements graphs, AFM and fluorescence microscopy images on other (ordered and disordered) nanotopographies and on the flat surface, steps of image processing for FAs analysis, schematic of the cantilever tip. (PDF)

All the original data related to this manuscript is within the depository of the University of Glasgow with doi: $10.5525 /$ gla.researchdata.332.

\section{AUTHOR INFORMATION}

\section{Corresponding Authors}

*marco.cantini@glasgow.ac.uk.

*manuel.salmeron-sanchez@glasgow.ac.uk.

\section{Author Contributions}

${ }^{\S}$ E.N.M. and M.C. contributed equally to this work.

Notes

The authors declare no competing financial interest.

\section{ACKNOWLEDGMENTS}

Financial support from ERC through HealInSynergy (306990) and MRC (MR/L022710/1) is acknowledged. EPSRC funding as part of the Doctoral Training Centre EP/F500424/1 is also acknowledged.

\section{REFERENCES}

(1) Dalby, M. J.; Childs, S.; Riehle, M. O.; Johnstone, H. J.; Affrossman, S.; Curtis, A. S. Fibroblast Reaction to Island Topography: Changes in Cytoskeleton and Morphology with Time. Biomaterials 2003, 24, 927-935.

(2) Curtis, A. S. G.; Dalby, M. J.; Gadegaard, N. Nanoprinting onto Cells. J. R. Soc., Interface 2006, 3, 393-398.

(3) Tsimbouri, P.; Gadegaard, N.; Burgess, K.; White, K.; Reynolds, P.; Herzyk, P.; Oreffo, R; Dalby, M. J. Nanotopographical Effects on Mesenchymal Stem Cell Morphology and Phenotype. J. Cell. Biochem. 2014, 115, 380-390.

(4) McMurray, R. J.; Gadegaard, N.; Tsimbouri, P. M.; Burgess, K. V.; McNamara, L. E.; T are, R.; Murawski, K.; Kingham, E.; Oreffo, R. O. C.; Dalby, M. J. Nanoscale Surfaces for the Long-Term Maintenance of Mesenchymal Stem Cell Phenotype and Multipotency. Nat. Mater. 2011, 10, 637-644.
(5) Dalby, M. J.; Gadegaard, N.; Tare, R.; Andar, A.; Riehle, M. O.; Herzyk, P.; Wilkinson, C. D. W.; Oreffo, R. O. C. The Control of Human Mesenchymal Cell Differentiation Using Nanoscale Symmetry and Disorder. Nat. Mater. 2007, 6, 997-1003.

(6) Curtis, A. S.; Varde, M. Control of Cell Behavior: Topological Factors. J. Natl. Cancer Inst. 1964, 33, 15-26.

(7) Curtis, A. S.; Gadegaard, N.; Dalby, M. J.; Riehle, M. O.; Wilkinson, C. D.; Aitchison, G. Cells React to Nanoscale Order and Symmetry in Their Surroundings. IEEE Trans. Nanobiosci. 2004, 3, $61-65$.

(8) González-García, C.; Sousa, S. R; Moratal, D.; Rico, P.; Salmerón-Sánchez, M. Effect of Nanoscale Topography on Fibronectin Adsorption, Focal Adhesion Size and Matrix Organisation. Colloids Surf., B 2010, 77, 181-190.

(9) Biggs, M. J.; Richards, R. G.; Gadegaard, N.; Wilkinson, C. D.; Dalby, M. J. The Effects of Nanoscale Pits on Primary Human Osteoblast Adhesion Formation and Cellular Spreading. J. Mater. Sci.: Mater. Med. 2007, 18, 399-404.

(10) Tsimbouri, P. M.; McMurray, R. J.; Burgess, K. V.; Alakpa, E. V.; Reynolds, P. M.; Murawski, K.; Kingham, E.; Oreffo, R. O.; Gadegaard, N.; Dalby, M. J. Using Nanotopography and Metabolomics to Identify Biochemical Effectors of Multipotency. ACS Nano 2012, 6, 1023910249.

(11) Dalby, M. J.; Gadegaard, N.; Oreffo, R. O. Harnessing Nanotopography and Integrin-Matrix Interactions to Influence Stem Cell Fate. Nat. Mater. 2014, 13, 558-569.

(12) Wood, M. A.; Bagnaninchi, P.; Dalby, M. J. The Beta Integrins and Cytoskeletal Nanoimprinting. Exp. Cell Res. 2008, 314, 927-935.

(13) Wang, N.; Butler, J. P.; Ingber, D. E. Mechanotransduction Across the Cell Surface and Through the Cytoskeleton. Science 1993, 260, 1124-1127.

(14) Ravichandran, R.; Liao, S.; Ng, C.; Chan, C. K.; Raghunath, M.; Ramakrishna, S. Effects of Nanotopography on Stem Cell Phenotypes. World J. Stem Cells 2009, 1, 55-66.

(15) Wang, N.; Naruse, K.; Stamenovic, D.; Fredberg, J. J.; Mijailovich, S. M.; Tolic-Norrelykke, I. M.; Polte, T.; Mannix, R.; Ingber, D. E. Mechanical Behavior in Living Cells Consistent with the Tensegrity Model. Proc. Natl. Acad. Sci. U. S. A. 2001, 98, 7765-7770.

(16) García, A. Interfaces to Control Cell-Biomaterial Adhesive Interactions. In Polymers for Regenerative Medicine; Werner, C., Ed.; Springer: Berlin, 2006; pp 171-190.

(17) Anselme, K.; Ponche, A.; Bigerelle, M. Relative Influence of Surface Topography and Surface Chemistry on Cell Response to Bone Implant Materials. Part 2: Biological Aspects. Proc. Inst. Mech. Eng., Part H 2010, 224, 1487-507.

(18) Rico, P.; Cantini, M.; Altankov, G.; Salmerón-Sánchez, M. Matrix Protein Interactions with Synthetic Surfaces. In Polymers in Regenerative Medicine: Biomedical Applications from Nano- to MacroStructures; Monleón Pradas, M., Vicent, M. J., Eds.; John Wiley \& Sons, Inc, 2014; pp 91-146.

(19) Planell, J.; Navarro, M.; Altankov, G.; Aparicio, C.; Engel, E.; Gil, J.; Ginebra, M.; Lacroix, D., Materials Surface Effects on Biological Interactions. In Advances in Regenerative Medicine: Role of Nanotechnology, and Engineering Principles; Shastri, V. P., Altankov, G., Lendlein, A., Eds.; Springer: Netherlands, 2010; pp 233-252.

(20) Roach, P.; Eglin, D.; Rohde, K.; Perry, C. C. Modern Biomaterials: A Review - Bulk Properties and Implications of Surface Modifications. J. Mater. Sci.: Mater. Med. 2007, 18, 1263-1277.

(21) Perez-Garnes, M.; Gonzalez-Garcia, C.; Moratal, D.; Rico, P.; Salmeron-Sanchez, M. Fibronectin Distribution on Demixed Nanoscale Topographies. Int. J. Artif. Organs 2011, 34, 54-63.

(22) Karakecili, A.; Messina, G. M.; Yurtsever, M. C.; Gumusderelioglu, M.; Marletta, G. Impact of Selective Fibronectin Nanoconfinement on Human Dental Pulp Stem Cells. Colloids Surf., B 2014, 123, 39-48.

(23) Hyun, Y. M.; Chung, H. L.; McGrath, J. L.; Waugh, R. E.; Kim, M. Activated Integrin VLA-4 Localizes to the Lamellipodia and Mediates T Cell Migration on VCAM-1. J. Immunol. 2009, 183, 359369. 
(24) Campbell, I. D.; Humphries, M. J. Integrin Structure, Activation, and Interactions. Cold Spring Harbor Perspect. Biol. 2011, 3, a004994.

(25) Berginski, M. E.; Gomez, S. M. The Focal Adhesion Analysis Server: a Web Tool for Analyzing Focal Adhesion Dynamics. F1000Research 2013, 2, 68.

(26) Vanterpool, F. A.; Cantini, M.; Seib, F. P.; Salmeron-Sanchez, M. A Material-Based Platform to Modulate Fibronectin Activity and Focal Adhesion Assembly. BioRes. Open Access 2014, 3, 286-296.

(27) Geiger, B.; Bershadsky, A.; Pankov, R.; Yamada, K. M. Transmembrane Crosstalk Between the Extracellular Matrix and the Cytoskeleton. Nat. Rev. Mol. Cell Biol. 2001, 2, 793-805.

(28) Biggs, M. J.; Richards, R. G.; Gadegaard, N.; Wilkinson, C. D.; Oreffo, R. O.; Dalby, M. J. The Use of Nanoscale Topography to Modulate the Dynamics of Adhesion Formation in Primary Osteoblasts and ERK/MAPK Signalling in STRO-1+ Enriched Skeletal Stem Cells. Biomaterials 2009, 30, 5094-5103.

(29) Curtis, A.; Wilkinson, C. Nantotechniques and Approaches in Biotechnology. Trends Biotechnol. 2001, 19, 97-101.

(30) Chen, W.; Villa-Diaz, L. G.; Sun, Y.; Weng, S.; Kim, J. K.; Lam, R. H.; Han, L.; Fan, R.; Krebsbach, P. H.; Fu, J. Nanotopography Influences Adhesion, Spreading, and Self-Renewal of Human Embryonic Stem Cells. ACS Nano 2012, 6, 4094-4103.

(31) Ji, L.; LaPointe, V. L.; Evans, N. D.; Stevens, M. M. Changes in Embryonic Stem Cell Colony Morphology and Early Differentiation Markers Driven by Colloidal Crystal Topographical Cues. Eur. Cells Mater. 2012, 23, 135-146.

(32) Lee, E. A.; Jung, G.; Im, S. G.; Hwang, N. S. Extracellular Matrix-Immobilized Nanotopographical Substrates for Enhanced Myogenic Differentiation. J. Biomed. Mater. Res., Part B 2015, 103, $1258-1266$.

(33) Vaz, R.; Martins, G. G.; Thorsteinsdottir, S.; Rodrigues, G. Fibronectin Promotes Migration, Alignment and Fusion in an in Vitro Myoblast Cell Model. Cell Tissue Res. 2012, 348, 569-578.

(34) Gallagher, J. O.; McGhee, K. F.; Wilkinson, C. D.; Riehle, M. O. Interaction of Animal Cells with Ordered Nanotopography. IEEE Trans. Nanobiosci. 2002, 1, 24-28.

(35) Dalby, M. J.; Berry, C. C.; Riehle, M. O.; Sutherland, D. S.; Agheli, H.; Curtis, A. S. Attempted Endocytosis of Nano-Environment Produced by Colloidal Lithography by Human Fibroblasts. Exp. Cell Res. 2004, 295, 387-394.

(36) Dalby, M. J.; Biggs, M. J.; Gadegaard, N.; Kalna, G.; Wilkinson, C. D.; Curtis, A. S. Nanotopographical Stimulation of Mechanotransduction and Changes in Interphase Centromere Positioning. J. Cell. Biochem. 2007, 100, 326-338.

(37) Biggs, M. J.; Richards, R. G.; Gadegaard, N.; Wilkinson, C. D.; Dalby, M. J. Regulation of Implant Surface Cell Adhesion: Characterization and Quantification of S-phase Primary Osteoblast Adhesions on Biomimetic Nanoscale Substrates. J. Orthop. Res. 2007, 25, 273282.

(38) Gadegaard, N.; Mosler, S.; Larsen, N. B. Biomimetic Polymer Nanostructures by Injection Molding. Macromol. Mater. Eng. 2003, $288,76-83$. 